Article

\title{
Conorms over anti fuzzy vector spaces
}

\section{Rasul Rasuli}

Department of Mathematics, Payame Noor University (PNU), Tehran, Iran.; rasulirasul@yahoo.com

Received: 14 September 2019; Accepted: 22 January 2020; Published: 1 May 2020.

\begin{abstract}
In this work, by using $t$-conorm $C$, we introduce anti fuzzy vector spaces and define sum, union, direct sum and normality of anti fuzzy vector spaces. We prove that sum, union, direct sum and normality of anti fuzzy vector spaces is also anti fuzzy vector space under $t$-conorm $C$. Moreover, we investigate linear transformations over anti fuzzy vector spaces (normal anti fuzzy vector spaces) under $t$-conorms and prove that image and pre image of them is also anti fuzzy vector space (normal anti fuzzy vector space) under $t$-conorms.
\end{abstract}

Keywords: Fuzzy set theory, vector spaces, anti fuzzy vector space, norms, direct sum, homomorphisms.

MSC: 03E72, 15A03, 47A30, 20K25, 20K30.

\section{Introduction}

$\mathbf{T}$ he concept of a fuzzy subset of a nonempty set was introduced by Zadeh in 1965 [1] as a function from a nonempty set $X$ into the unit real interval $I=[0,1]$. Rosenfeld [2] extended the idea of Zadeh to the theory of groupoids and groups. The concepts of fuzzy field and fuzzy linear space over fuzzy field were introduced by Nanda [3]. In 1977, Katsaras and Liu [4] studied the notion of fuzzy vector subspaces over the field of real or complex numbers. After Katsaras and Liu [4], many scholars investigated properties and characteristics of fuzzy vector subspaces [5-8]. Hohle [9] and Alsina et al., [10] introduced T-norm in fuzzy set theory and proved that the $T$-norm can be used for the intersection of fuzzy sets. Since then, many researchers presented various types of $T$-norms for particular purposes [11,12].

The author by using norms, investigated some properties of fuzzy algebraic structures [13-18]. This work is an attempt to study purely algebraic properties of anti fuzzy vector spaces under $t$-conorms. In this paper, the author introduce anti fuzzy vector spaces under $t$-conorms and investigate relationship between anti fuzzy vector spaces under $t$-conorms and vector subspaces. Also the author introduce sum, union, direct sum of anti fuzzy vector spaces under $t$-conorms and prove that sum, union, direct sum of them is also anti fuzzy vector spaces under $t$-conorms. Later, the author define normal anti fuzzy vector spaces under $t$-conorm and obtain some results about them. Finally, the author investigate linear transformations over anti fuzzy vector spaces (normal anti fuzzy vector spaces) under $t$-conorms and show that image and pre image of them is also anti fuzzy vector space (normal anti fuzzy vector spaces) under $t$-conorms.

\section{Preliminaries and main result}

\subsection{Preliminaries}

This section recalls some of the fundamental concepts and definitions necessary for this paper.

Definition 1. ([19]) A vector space or a linear space consists of the following:

(1) a field $\mathbb{F}$ of scalars.

(2) a set $V$ of objects called vectors.

(3) a rule (or operation) called vector addition: associates with each pair of vectors $\alpha, \beta \in V ; \alpha+\beta \in V$, called the sum of $\alpha$ and $\beta$ in such a way that

(a) addition is commutative $\alpha+\beta=\beta+\alpha$,

(b) addition is associative $\alpha+(\beta+\gamma)=(\alpha+\beta)+\gamma$,

(c) there is a unique vector $0 \in V$, called the zero vector, such that $\alpha+0=\alpha$ for all $\alpha \in V$,

(d) for each vector $\alpha \in V$ there is a unique vector $(-\alpha)$ in $V$ such that $\alpha+(-\alpha)=0$, 
(e) a rule (or operation), called scalar multiplication, associates with each scalar $c$ in $\mathbb{F}$ and a vector $\alpha$ in $V$, a vector $c \bullet \alpha$ in $V$, called the product of $c$ and $\alpha$, in such a way that $1 \bullet \alpha=\alpha,\left(c_{1} \bullet c_{2}\right) \bullet \alpha=$ $c_{1} \bullet\left(c_{2} \bullet \alpha\right), c \bullet(\alpha+\beta)=c \bullet \alpha+c \bullet \beta, \quad\left(c_{1}+c_{2}\right) \bullet \alpha=\left(c_{1} \bullet \alpha\right)+\left(c_{2} \bullet \alpha\right)$ for $\alpha, \beta \in V$ and $c_{,} c_{1}, c_{2} \in F$. It is important to note as the definition states that a vector space is a composite object consisting of a field, a set of a vector space and two operations with certain special properties. The same set of vectors may be part of a number of distinct vectors. We simply use the notation, $V$ a vector space over the field $\mathbb{F}$ and call elements of $V$ as vectors.

Throughout this section, $\mathbb{F}$ is any field of characteristic zero.

Example 1. Let $V=\mathbb{R} \times \mathbb{R} \times \mathbb{R}$. Then $V$ is a vector space over $\mathbb{R}$ or $\mathbb{Q}$ but $V$ is not a vector space over the complex field $\mathbb{C}$.

Definition 2. [19] Let $V$ be a vector space over the field $\mathbb{F}$. A subspace of $V$ is a subset $W$ of $V$ which is itself a vector space over $\mathbb{F}$ with the same operations of vector addition and scalar multiplication as on $V$.

We have the following nice characterization theorem for subspaces.

Theorem 1. [19] Let $W$ be a non-empty subset of a vector $V$ over the field $\mathbb{F}$. Then $W$ is a subspace of $V$ if and only if for each pair $\alpha, \beta \in W$ and each scalar $c \in \mathbb{F}$ the vector $c \alpha+\beta \in W$.

Example 2. Let $M_{n \times n}=\left\{\left(a_{i j}\right) \mid a_{i j} \in \mathbb{Q}\right\}$ be the vector space over $\mathbb{Q}$. Let $D_{n \times n}=\left\{\left(a_{i i}\right) \mid a_{i i} \in \mathbb{Q}\right\}$ be the set of all diagonal matrices with entries from $\mathbb{Q}$. Then $D_{n \times n}$ is a subspace of $M_{n \times n}$.

Definition 3. [19] Let $V$ and $W$ be two vector spaces over the field of $\mathbb{F}$. A map $f: V \rightarrow W$ is called a linear transformation if $f(a x+y)=a f(x)+f(y)$ for all $x, y \in V$ and $a \in \mathbb{F}$.

Definition 4. [20] Let $X$ be a non-empty set. A fuzzy subset $\mu$ of $X$ is a function $\mu: X \rightarrow[0,1]$.

The set of all fuzzy subsets of $X$ is denoted by $[0,1]^{X}$

Definition 5. [21] A $t$-conorm $C$ is a function $C:[0,1] \times[0,1] \rightarrow[0,1]$ having the following four properties:

(C1) $C(x, 0)=x$,

(C2) $C(x, y) \leq C(x, z)$ if $y \leq z$,

(C3) $C(x, y)=C(y, x)$,

(C4) $C(x, C(y, z))=C(C(x, y), z)$,

for all $x, y, z \in[0,1]$.

Corollary 2. [21] Let C be a C-conorm, then for all $x \in[0,1]$, we have

(1) $C(x, 1)=1$,

(2) $C(0,0)=0$.

Example 3. [21]

(1) Standard union $t$-conorm $C_{m}(x, y)=\max \{x, y\}$.

(2) Bounded sum $t$-conorm $C_{b}(x, y)=\min \{1, x+y\}$.

(3) Algebraic sum $t$-conorm $C_{p}(x, y)=x+y-x y$.

(4) Drastic $T$-conorm $C_{D}(x, y)= \begin{cases}y & \text { if } x=0 \\ x & \text { if } y=0 \\ 1 & \text { otherwise. }\end{cases}$

(5) Nilpotent maximum $T$-conorm: $C_{n M}(x, y)=\left\{\begin{aligned} \max \{x, y\} & \text { if } x+y<1 \\ 1 & \text { otherwise. }\end{aligned}\right.$

(6) Einstein sum (compare the velocity-addition formula under special relativity) $C_{\mathrm{H}_{2}}(x, y)=\frac{x+y}{1+x y}$. Note that all $t$-conorms are bounded by the maximum and the drastic t-conorm: $C_{\max }(x, y) \leq C(x, y) \leq$ $C_{D}(x, y)$ for any $t$-conorm $C$ and all $x, y \in[0,1]$. 
Recall that $t$-conorm $C$ is idempotent if for all $x \in[0,1]$, we have that $C(x, x)=x$.

Lemma 1. [21] Let $C$ be a t-conorm, then $C(C(x, y), C(w, z))=C(C(x, w), C(y, z))$, for all $x, y, w, z \in[0,1]$.

\section{Anti fuzzy vector spaces under $t$-conorms}

Throughout this section, $V$ is a vector space on a field $\mathbb{F}$ unless otherwise specified.

Definition 6. A fuzzy set $\mu: V \rightarrow[0,1]$ is called a anti fuzzy vector space of $V$ under $t$-conorm $C$, if for all $x, y \in V$ and $a \in \mathbb{F}$ the following conditions hold:

(1) $\mu(x+y) \leq C(\mu(x), \mu(y))$,

(2) $\mu(-x) \leq \mu(x)$,

(3) $\mu(a x) \leq \mu(x)$.

The set of all fuzzy vector spaces of $V$ under $t$-conorm $C$ is denoted by $A F C(V)$.

Corollary 3. Let $\mu \in A F C(V)$, then $\mu(-x)=\mu(x)$ for all $x \in V$.

Proof. Let $x \in V$ then $\mu(-x) \leq \mu(x)=\mu(-(-x)) \leq \mu(-x)$. Hence $\mu(-x)=\mu(x)$.

Example 4. Let $V=\mathbb{R} \times \mathbb{R} \times \mathbb{R}$ be a vector space over a field $\mathbb{F}=\mathbb{R}$. Define $\mu: \mathbb{R} \times \mathbb{R} \times \mathbb{R} \rightarrow[0,1]$ as

$$
\mu(x, y, z)= \begin{cases}0.40 & (x, y, z) \in\{(0, y, 0) \mid y \in \mathbb{R}\} \\ 0.55 & \text { otherwise }\end{cases}
$$

Let $C(a, b)=C_{p}(a, b)=a+b-a b$ for all $a, b \in[0,1]$, then $\mu \in A F C(V)$.

Theorem 4. Let $V$ be a subspace over field $\mathbb{F}$ and $W$ be a subset of $V$ and $\mu: W \rightarrow\{0,1\}$ be the characteristic function such that $W$ be a subspace of $V$, then $\mu \in A F C(V)$.

Proof. Let $x, y \in V$ and we investigate the following conditions:

(1) If $x, y \in W$, then $x+y \in W$ and we have $\mu(x+y)=1 \leq 1=C(1,1)=C(\mu(x), \mu(y))$.

(2) If $x \notin W$ and $y \in W$, then $x+y \notin W$ and then $\mu(x+y)=0 \leq 0=C(0, \mu(y))=C(\mu(x), \mu(y))$.

(3) Finally, if $x, y \notin W$, then $\mu(x+y)=0 \leq 0=C(0,0)=C(\mu(x), \mu(y))$.

Thus from $(1)-(3)$, we have $\mu(x+y) \leq C(\mu(x), \mu(y))$. Also, we have that the following conditions:

(4) If $x \in W$, then $-x \in W$ and then $\mu(-x)=1 \leq 1=\mu(x)$.

(5) If $x \notin W$, then $-x \notin W$ and then $\mu(-x)=0 \leq 0=\mu(x)$.

Thus from $(4)-(5)$, we have that $\mu(-x) \leq \mu(x)$. Now, let $a \in \mathbb{F}$ :

(6) If $x \in W$, then $a x \in W$ and so $\mu(a x)=1 \leq 1=\mu(x)$.

(7) If $x \notin W$, then $a x \notin W$ so $\mu(a x)=0 \leq 0=\mu(x)$.

From (6) and (7), we obtain $\mu(a x) \leq \mu(x)$. Therefore, the above conditions gives $\mu \in A F C(V)$.

Theorem 5. Let $\mu \in A F C(V)$, then $W=\{x \mid x \in V: \mu(x)=0\}$ is either empty or is a subspace of $V$.

Proof. Let $x, y \in W$ and $a \in \mathbb{F}$. Then $\mu(a x+y) \leq C(\mu(a x), \mu(y)) \leq C(\mu(x), \mu(y))=C(0,0)=0$ and $\mu(a x+$ $y)=0$ which means that $a x+y \in W$ and by Theorem 1 , we get $W$ is a subspace of $V$.

Theorem 6. If $\mu \in A F C(V)$ and $C$ be an idempotent $t$-conorm, then $\mu(0) \leq \mu(x)$ for all $x \in V$.

Proof. As $\mu \in A F C(V)$ so $\mu(0)=\mu(x-x)=\mu(x+(-x)) \leq C(\mu(x), \mu(-x)) \leq C(\mu(x), \mu(x))=\mu(x)$. Thus $\mu(0) \leq \mu(x)$.

Theorem 7. Let $\mu \in A F C(V)$ and $C$ be an idempotent $t$-conorm. Then $W=\{x \mid x \in V: \mu(x)=\mu(0)\}$ is either empty or is a subspace of $V$. 
Proof. Let $x, y \in W$ and $a \in \mathbb{F}$. Then by by Theorem 6, we have $\mu(a x+y) \leq C(\mu(a x), \mu(y)) \leq C(\mu(x), \mu(y))=$ $C(\mu(0), \mu(0))=\mu(0) \leq \mu(a x+y)$. Thus $\mu(a x+y)=\mu(0)$ and $a x+y \in W$. Hence Theorem 1 gives us that $W$ is a subspace of $V$.

Theorem 8. Let $\mu \in A F C(V)$ and $C$ be an idempotent $t$-conorm. If $\mu(x-y)=\mu(0)$, then $\mu(x)=\mu(y)$ for all $x, y \in V$.

Proof. Let $\mu \in A F C(V)$ then $\mu(x)=\mu(x-y+y) \leq C(\mu(x-y), \mu(y)) \leq C(\mu(0), \mu(y)) \leq C(\mu(y), \mu(y))$ $=\mu(y)=\mu(y+x-x)=\mu(-(x-y)+x) \leq C(\mu(-(x-y)), \mu(x)) \leq C(\mu(x-y), \mu(x))=C(\mu(0), \mu(x))$ $\leq C(\mu(x), \mu(x))=\mu(x)$. Therefore $\mu(x)=\mu(y)$.

Theorem 9. Let $C$ be an idempotent t-conorm. Then $\mu \in A F C(V)$ if and only if $\mu(x-y) \leq C(\mu(x), \mu(y))$ and $\mu(a x) \leq \mu(x)$ for all $x, y \in V$ and $a \in \mathbb{F}$.

Proof. Let $\mu \in A F C(V)$, then $\mu(x-y)=\mu(x+(-y)) \leq C(\mu(x), \mu(-y)) \leq C(\mu(x), \mu(y))$.

Conversely, let $\mu(x-y) \leq C(\mu(x), \mu(x))$ and $\mu(a x) \leq \mu(x)$. Then

$$
\mu(0)=\mu(x-x) \leq C(\mu(x), \mu(x))=\mu(x),
$$

and

$$
\mu(-x)=\mu(0-x) \leq C(\mu(0), \mu(x)) \leq C(\mu(x), \mu(x))=\mu(x) .
$$

Also

$$
\mu(x+y)=\mu(x-(-y)) \leq C(\mu(x), \mu(-y)) \leq C(\mu(x), \mu(y)),
$$

therefore, $\mu \in A F C(V)$.

Theorem 10. Let $\mu: V \rightarrow[0,1]$ be a fuzzy set. If $\mu(0)=0, \mu(x-y) \leq C(\mu(x), \mu(y))$ and $\mu(a x) \leq \mu(x)$, then $\mu \in A F C(V)$ for all $x, y \in V$ and $a \in \mathbb{F}$.

Proof. We have $\mu(-x)=\mu(0-x) \leq C(\mu(0), \mu(x))=C(0, \mu(x))=\mu(x)$. Also $\mu(x+y)=\mu(x-(-y)) \leq$ $C(\mu(x), \mu(-y)) \leq C(\mu(x), \mu(y))$. Thus $\mu \in A F C(V)$.

Theorem 11. Let $\mu \in A F C(V)$ and $\mu(x-y)=0$, then $\mu(x)=\mu(y)$ for all $x, y \in V$.

Proof. Let $x, y \in V$, then by using Corollary 3, we have $\mu(x)=\mu(x-y+y) \leq C(\mu(x-y), \mu(y))=$ $T(0, \mu(y))=\mu(y)=\mu(-y)=\mu(x-x-y)=\mu(x-y-x) \leq C(\mu(x-y), \mu(-x))=C(0, \mu(-x))=\mu(-x)=$ $\mu(x)$. Therefore $\mu(x)=\mu(y)$.

Theorem 12. Let $\mu \in A F C(V)$ and $\mu(x-y)=1$. Then either $\mu(x)=1$ or $\mu(y)=1$ for all $x, y \in V$.

Proof. Let $x, y \in V$ then $1=\mu(x-y)=\mu(x+(-y)) \leq C(\mu(x), \mu(-y)) \leq C(\mu(x), \mu(y))$ which implies that either $\mu(x)=1$ or $\mu(y)=1$.

Theorem 13. Let $\mu \in A F C(V)$ and $\mu(x)<\mu(y)$ for some $x, y \in V$. If C be an idempotent $t$-conorm, then $\mu(x+y)=$ $\mu(y)$ for all $x, y \in V$.

Proof. Let $\mu \in A F C(V)$ and $\mu(x)<\mu(y)$ which means that $\mu(x)<\mu(x+y)$ for all $x, y \in V$. Then $\mu(x+y) \leq$ $C(\mu(x), \mu(y)) \leq C(\mu(y), \mu(y))=\mu(y)=\mu(x+y-x) \leq C(\mu(x+y), \mu(-x)) \leq C(\mu(x+y), \mu(x)) \leq C(\mu(x+$ $y), \mu(x+y))=\mu(x+y)$. Thus $\mu(x+y)=\mu(y)$.

Theorem 14. Let $\mu \in A F C(V)$ and $\mu(x)>\mu(y)$ for some $x, y \in V$. If $C$ be an idempotent $t$-conorm, then $\mu(x+y)=$ $\mu(x)$ for all $x, y \in V$.

Proof. It is trivial.

Theorem 15. Let $\mu \in A F C(V)$ and $C$ be an idempotent $t$-conorm. If $\mu(x) \neq \mu(y)$, then $\mu(x+y)=C(\mu(x), \mu(y))$ for all $x, y \in V$. 
Proof. (1) If $\mu(x)<\mu(y)$, then $\mu(y)=C(\mu(x), \mu(y))$ for all $x, y \in V$. Now from Theorem 13, we have $\mu(x+y)=\mu(y)$ and then $\mu(x+y)=\mu(y)=C(\mu(x), \mu(y))$.

(2) If $\mu(x)>\mu(y)$, then $\mu(x)=C(\mu(x), \mu(y))$ for all $x, y \in V$. Now from Theorem 14 , we have $\mu(x+y)=$ $\mu(x)$ and then $\mu(x+y)=\mu(x)=C(\mu(x), \mu(y))$.

The conditions (1) and (2) gives us: If $\mu(x) \neq \mu(y)$, then $\mu(x+y)=C(\mu(x), \mu(y))$ for all $x, y \in V$.

Theorem 16. Let $\mu \in A F C(V)$ and $C$ be an idempotent $t$-conorm. Then $\mu(x-y)=\mu(y)$ if and only if $\mu(x)=\mu(0)$ for all $x, y \in V$.

Proof. Let $\mu(x-y)=\mu(y)$ and $y=0$ we have $\mu(x)=\mu(0)$.

Conversely, suppose that $\mu(x)=\mu(0)$. From Theorem 6, we have $\mu(x)=\mu(0) \leq \mu(x-y)$ and $\mu(x)=$ $\mu(0) \leq \mu(-y)$.

Therefore, $\mu(x-y)=\mu(x+(-y)) \leq C(\mu(x), \mu(-y))=C(\mu(0), \mu(-y)) \leq C(\mu(-y), \mu(-y))=\mu(-y)=$ $\mu(x-y-x)=\mu(x-y+(-x)) \leq \leq C(\mu(x-y), \mu(-x)) \leq C(\mu(x-y), \mu(x))=C(\mu(x-y), \mu(0)) \leq C(\mu(x-$ $y), \mu(x-y))=\mu(x-y)$.

Hence, $\mu(x-y)=\mu(-y)$ and by Corollary 3 , we have $\mu(y)=\mu(-y)=\mu(x-y)$.

Theorem 17. Let $\mu \in A F C(V)$ and $f:[0, \mu(0)] \rightarrow[0,1]$ be a decreasing map. Define a fuzzy set $\mu^{f}: V \rightarrow[0,1]$ by $\mu^{f}(x)=f(\mu(x))$. Then $\mu^{f} \in A F C(V)$.

Proof. Let $x, y \in V$ and $a \in \mathbb{F}$. Then

(1) $\mu^{f}(x+y)=f(\mu(x+y)) \leq f(C(\mu(x), \mu(y)))=C(f(\mu(x)), f(\mu(y)))=C\left(\mu^{f}(x), \mu^{f}(y)\right)$.

(2) $\mu^{f}(-x)=f(\mu(-x)) \leq f(\mu(x))=\mu^{f}(x)$.

(3) $\mu^{f}(a x)=f(\mu(a x)) \leq f(\mu(x))=\mu^{f}(x)$.

Therefore $\mu^{f} \in \operatorname{AFC}(V)$.

\section{Sum, union, direct sum and nomality of anti fuzzy vector spaces under $t$-conorms}

Definition 7. Let $\mu_{1}, \mu_{2} \in A F C(V)$. The sum of $\mu_{1}$ and $\mu_{2}$ is defined as follows:

$$
\left(\mu_{1}+\mu_{2}\right)(x):=\sup \left\{C\left(\mu_{1}(y), \mu_{2}(z)\right) \mid x=y+z \in V\right\} .
$$

Proposition 1. Let $\mu_{1}, \mu_{2} \in A F C(V)$ and $C$ be idempotent t-conorm. Then $\left(\mu_{1}+\mu_{2}\right) \in A F C(V)$.

Proof. (1) Let $x_{1}, x_{2}, y_{1}, y_{2}, z_{1}, z_{2} \in V$. Then using Lemma 1 , we have

$$
\begin{aligned}
\left(\mu_{1}+\mu_{2}\right)\left(x_{1}+x_{2}\right) & =\sup \left\{C\left(\mu_{1}\left(y_{1}+y_{2}\right), \mu_{2}\left(z_{1}+z_{2}\right)\right) \mid x_{1}+x_{2}=y_{1}+y_{2}+z_{1}+z_{2}\right\} \\
& \leq \sup \left\{C\left(C\left(\mu_{1}\left(y_{1}\right), \mu_{1}\left(y_{2}\right)\right), C\left(\mu_{2}\left(z_{1}\right), \mu_{2}\left(z_{2}\right)\right)\right) \mid x_{1}+x_{2}=y_{1}+z_{1}+\left(y_{2}+z_{2}\right)\right\} \\
& =\sup \left\{C\left(C\left(\mu_{1}\left(y_{1}\right), \mu_{2}\left(z_{1}\right)\right), C\left(\mu_{1}\left(y_{2}\right), \mu_{2}\left(z_{2}\right)\right)\right) \mid x_{1}+x_{2}=y_{1}+z_{1}+\left(y_{2}+z_{2}\right)\right\} \\
& =C\left(\sup \left\{C\left(\mu_{1}\left(y_{1}\right), \mu_{2}\left(z_{1}\right)\right) \mid x_{1}=y_{1}+z_{1}\right\}, \sup \left\{C\left(\mu_{1}\left(y_{2}\right), \mu_{2}\left(z_{2}\right)\right) \mid x_{2}=y_{2}+z_{2}\right\}\right) \\
& =C\left(\left(\mu_{1}+\mu_{2}\right)\left(x_{1}\right),\left(\mu_{1}+\mu_{2}\right)\left(x_{2}\right)\right) .
\end{aligned}
$$

(2) Let $x, y, z \in V$, then

$$
\begin{aligned}
\left(\mu_{1}+\mu_{2}\right)(-x) & =\sup \left\{C\left(\mu_{1}(-y), \mu_{2}(-z)\right) \mid-x=-y+(-z)\right\} \\
& \leq \sup \left\{C\left(\mu_{1}(y), \mu_{2}(z)\right) \mid x=y+z\right\} \\
& =\left(\mu_{1}+\mu_{2}\right)(x) .
\end{aligned}
$$

(3) Let $x, y, z \in V$ and $a \in \mathbb{F}$, then

$$
\begin{aligned}
\left(\mu_{1}+\mu_{2}\right)(a x) & =\sup \left\{C\left(\mu_{1}(a y), \mu_{2}(a z)\right) \mid a x=a y+a z\right\} \\
& \leq \sup \left\{C\left(\mu_{1}(y), \mu_{2}(z)\right) \mid x=y+z\right\} \\
& =\left(\mu_{1}+\mu_{2}\right)(x)
\end{aligned}
$$

Thus $\left(\mu_{1}+\mu_{2}\right) \in A F C(V)$. 
Definition 8. Let $\mu_{1}, \mu_{2} \in A F C(V)$ then by union of fuzzy subsets $\mu_{1}$ and $\mu_{1}$ with respect to a $t$-conorm $C$, we mean the fuzzy subset $\mu=\mu_{1} \cup \mu_{2}$ such that for any $x \in V, \mu(x)=\left(\mu_{1} \cup \mu_{2}\right)(x)=C\left(\mu_{1}(x), \mu_{2}(x)\right)$.

Theorem 18. If $\mu_{1}, \mu_{2} \in A F C(V)$, then $\mu_{1} \cup \mu_{2} \in A F C(V)$.

Proof. Let $x, y \in V$ and $a \in \mathbb{F}$. Then by using Lemma 1, we have

(1) $\left(\mu_{1} \cup \mu_{2}\right)(x+y)=C\left(\mu_{1}(x+y), \mu_{2}\right)(x+y) \leq C\left(C\left(\mu_{1}(x), \mu_{1}(y)\right), C\left(\mu_{2}(x), \mu_{2}(y)\right)\right)$ $=C\left(C\left(\mu_{1}(x), \mu_{2}(x)\right), C\left(\mu_{1}(y), \mu_{2}(y)\right)\right)=C\left(\left(\mu_{1} \cup \mu_{2}\right)(x),\left(\mu_{1} \cup \mu_{2}\right)(y)\right)$.

(2) $\left(\mu_{1} \cup \mu_{2}\right)(-x)=C\left(\mu_{1}(-x), \mu_{2}(-x)\right) \leq C\left(\mu_{1}(x), \mu_{2}(x)\right)=\left(\mu_{1} \cup \mu_{2}\right)(x)$.

(3) $\left(\mu_{1} \cup \mu_{2}\right)(a x)=C\left(\mu_{1}(a x), \mu_{2}(a x)\right) \leq C\left(\mu_{1}(x), \mu_{2}(x)\right)=\left(\mu_{1} \cup \mu_{2}\right)(x)$.

Thus $\mu_{1} \cup \mu_{2} \in A F C(V)$.

Definition 9. Let $\left\{\mu_{i}\right\}_{i \in I}$ be a family of fuzzy subspaces of $V$ under $t$-conorm $C$. Then their union $\mu=\cup_{i \in I} \mu_{i}$ : $V \rightarrow[0,1]$ is defined by $\mu(x)=\sup _{i \in I} \mu_{i}(x)$ for all $x \in V$.

Theorem 19. Let $\left\{\mu_{i}\right\}_{i \in I} \subseteq A F C(V)$, then $\mu=\cup_{i \in I} \mu_{i} \in A F C(V)$.

Proof. Let $x, y \in V$ and $a \in \mathbb{F}$. Then

(1) $\mu(x+y)=\sup _{i \in I} \mu_{i}(x+y) \leq \sup _{i \in I} C\left(\mu_{i}(x), \mu_{i}(y)\right)=C\left(\sup _{i \in I} \mu_{i}(x), \sup _{i \in I} \mu_{i}(y)\right)=C(\mu(x), \mu(y))$.

(2) $\mu(-x)=\sup _{i \in I} \mu_{i}(-x) \leq \sup _{i \in I} \mu_{i}(x)=\mu(x)$.

(3) $\mu(a x)=\sup _{i \in I} \mu_{i}(a x) \leq \sup _{i \in I} \mu_{i}(x)=\mu(x)$.

Therefore $\mu=\cup_{i \in I} \mu_{i} \in A F C(V)$.

Definition 10. For $\mu_{1} \in A F C\left(V_{1}\right)$ and $\mu_{2} \in A F C\left(V_{2}\right)$, the direct sum of $\mu_{1}$ and $\mu_{2}$ is denoted by $\mu_{1} \oplus \mu_{2}$ : $V_{1} \oplus V_{2} \rightarrow[0,1]$ and is defined by $\left(\mu_{1} \oplus \mu_{2}\right)\left(x_{1}, x_{2}\right)=C\left(\mu_{1}\left(x_{1}\right), \mu_{2}\left(x_{2}\right)\right)$ for all $x_{1} \in V_{1}$ and $x_{2} \in V_{2}$.

Theorem 20. Let $\mu_{1} \in A F C\left(V_{1}\right)$ and $\mu_{2} \in A F C\left(V_{2}\right)$, then $\mu_{1} \oplus \mu_{2} \in A F C\left(V_{1} \oplus V_{2}\right)$.

Proof. Let $\left(x_{1}, y_{1}\right),\left(x_{2}, y_{2}\right) \in V_{1} \oplus V_{2}$ and $a \in \mathbb{F}$. Then by using Lemma 1 , we have

(1) $\left(\mu_{1} \oplus \mu_{2}\right)\left(\left(x_{1}, y_{1}\right)+\left(x_{2}, y_{2}\right)\right)=\left(\mu_{1} \oplus \mu_{2}\right)\left(x_{1}+x_{2}, y_{1}+y_{2}\right)=C\left(\mu_{1}\left(x_{1}+x_{2}\right), \mu_{2}\left(y_{1}+y_{2}\right)\right)$ $\leq C\left(C\left(\mu_{1}\left(x_{1}\right), \mu_{1}\left(x_{2}\right)\right), C\left(\mu_{2}\left(y_{1}\right), \mu_{2}\left(y_{2}\right)\right)\right)=C\left(C\left(\mu_{1}\left(x_{1}\right), \mu_{2}\left(y_{1}\right)\right), C\left(\mu_{1}\left(x_{2}\right), \mu_{2}\left(y_{2}\right)\right)\right)$ $=C\left(\left(\mu_{1} \oplus \mu_{2}\right)\left(x_{1}, y_{1}\right),\left(\mu_{1} \oplus \mu_{2}\right)\left(x_{2}, y_{2}\right)\right)$.

(2) $\left(\mu_{1} \oplus \mu_{2}\right)\left(-\left(x_{1}, y_{1}\right)\right)=\left(\mu_{1} \oplus \mu_{2}\right)\left(-x_{1},-y_{1}\right)=C\left(\mu_{1}\left(-x_{1}\right), \mu_{2}\left(-y_{1}\right)\right) \leq C\left(\mu_{1}\left(x_{1}\right), \mu_{2}\left(y_{1}\right)\right)$ $=\left(\mu_{1} \oplus \mu_{2}\right)\left(x_{1}, y_{1}\right)$.

(3) $\left(\mu_{1} \oplus \mu_{2}\right)\left(a\left(x_{1}, y_{1}\right)\right)=\left(\mu_{1} \oplus \mu_{2}\right)\left(a x_{1}, a y_{1}\right)=C\left(\mu_{1}\left(a x_{1}\right), \mu_{2}\left(a y_{1}\right)\right) \leq C\left(\mu_{1}\left(x_{1}\right), \mu_{2}\left(y_{1}\right)\right)$ $=\left(\mu_{1} \oplus \mu_{2}\right)\left(x_{1}, y_{1}\right)$.

Then $\mu_{1} \oplus \mu_{2} \in A F C\left(V_{1} \oplus V_{2}\right)$.

Theorem 21. Let $C$ be an idempotent $t$-conorm and $0_{V_{1}}$ and $0_{V_{2}}$ are identity elements of $V_{1}$ and $V_{2}$, respectively. If $\mu_{1} \oplus \mu_{2} \in \operatorname{AFC}\left(V_{1} \oplus V_{2}\right)$, then $\left(\mu_{1} \oplus \mu_{2}\right)\left(0_{V_{1}}, 0_{V_{2}}\right) \leq\left(\mu_{1} \oplus \mu_{2}\right)(x, y)$ for all $(x, y) \in V_{1} \oplus V_{2}$.

Proof. Let $(x, y) \in V_{1} \oplus V_{2}$. Then by Theorem 6, we have $\left(\mu_{1} \oplus \mu_{2}\right)\left(0_{V_{1}}, 0_{V_{2}}\right)=C\left(\mu_{1}\left(0_{V_{1}}\right), \mu_{2}\left(0_{V_{2}}\right)\right) \leq$ $C\left(\mu_{1}(x), \mu_{2}(y)\right)=\left(\mu_{1} \oplus \mu_{2}\right)(x, y)$.

Theorem 22. Let $\mu_{1}: V_{1} \rightarrow[0,1]$ and $\mu_{2}: V_{2} \rightarrow[0,1]$ are two fuzzy subsets of the vector spaces $V_{1}$ and $V_{2}$, respectively and $0_{V_{1}}$ and $0_{V_{2}}$ are the identity elements of $V_{1}$ and $V_{2}$, respectively. Let $C$ be an idempotent $t$-conorm and $\mu_{1} \oplus \mu_{2} \in A F C\left(V_{1} \oplus V_{2}\right)$, then at least one of the following two statements must hold:

(1) $\mu_{2}\left(0_{V_{2}}\right) \leq \mu_{1}(x)$ for all $x \in V_{1}$.

(2) $\mu_{1}\left(0_{V_{1}}\right) \leq \mu_{2}(y)$ for all $y \in V_{2}$.

Proof. Let $\mu_{1} \oplus \mu_{2} \in A F C\left(V_{1} \oplus V_{2}\right)$. Suppose on contrary, none of the statement (1) and (2) holds. Then we can find $z \in V_{1}$ and $t \in V_{2}$ such that $\mu_{1}(z)<\mu_{2}\left(0_{V_{2}}\right)$ and $\mu_{2}(t)<\mu_{1}\left(0_{V_{1}}\right)$. Then $\left(\mu_{1} \oplus \mu_{2}\right)(z, t)=$ $C\left(\mu_{1}(z), \mu_{2}(t)\right)<C\left(\mu_{2}\left(0_{V_{2}}\right), \mu_{1}\left(0_{V_{1}}\right)\right)=C\left(\mu_{1}\left(0_{V_{1}}\right), \mu_{2}\left(0_{V_{2}}\right)\right)=\left(\mu_{1} \oplus \mu_{2}\right)\left(0_{V_{1}}, 0_{V_{2}}\right)$. 
Now from Theorem 6, we get $\mu_{1} \oplus \mu_{2} \notin A F C\left(V_{1} \oplus V_{2}\right)$, a contradiction. Therefore, either $\mu_{2}\left(0_{V_{2}}\right) \leq \mu_{1}(x)$ for all $x \in V_{1}$ or $\mu_{1}\left(0_{V_{1}}\right) \leq \mu_{2}(y)$ for all $y \in V_{2}$.

Theorem 23. Let $\mu_{1}: V_{1} \rightarrow[0,1]$ and $\mu_{2}: V_{2} \rightarrow[0,1]$ are two fuzzy subsets of the vector spaces $V_{1}$ and $V_{2}$, respectively and $0_{V_{1}}$ and $0_{V_{2}}$ are the identity elements of $V_{1}$ and $V_{2}$, respectively. Let $C$ be an idempotent $t$-conorm and $\mu_{1} \oplus \mu_{2} \in \operatorname{AFC}\left(V_{1} \oplus V_{2}\right)$, then the following statements holds:

(1) if $\mu_{1}(x) \geq \mu_{2}\left(0_{V_{2}}\right)$ for all $x \in V_{1}$, then $\mu_{1} \in A F C\left(V_{1}\right)$;

(2) if $\mu_{2}(x) \geq \mu_{1}\left(0_{V_{1}}\right)$ for all $x \in V_{2}$, then $\mu_{2} \in A F C\left(V_{2}\right)$;

(3) either $\mu_{1} \in A F C\left(V_{1}\right)$ or $\mu_{2} \in A F C\left(V_{2}\right)$.

Proof. (1) Let $x, y \in V_{1}, a \in \mathbb{F}$ and $\mu_{1} \oplus \mu_{2} \in A F C\left(V_{1} \oplus V_{2}\right)$. We will prove $\mu_{1} \in A F C\left(V_{1}\right)$. As $\mu_{1}(x) \geq \mu_{2}\left(0_{V_{2}}\right)$, so $\mu_{1}(x+y) \geq \mu_{2}\left(0_{V_{2}}\right)$ for all $x, y \in V_{1}$. Now $\mu_{1}(x+y)=C\left(\mu_{1}(x+y), \mu_{2}\left(0_{V_{2}}\right)\right)=$ $C\left(\mu_{1}(x+y), \mu_{2}\left(0_{V_{2}}+0_{V_{2}}\right)\right)=\left(\mu_{1} \oplus \mu_{2}\right)\left(x+y, 0_{V_{2}}+0_{V_{2}}\right)=\left(\mu_{1} \oplus \mu_{2}\right)\left(\left(x, 0_{V_{2}}\right)+\left(y, 0_{V_{2}}\right)\right) \leq C\left(\left(\mu_{1} \oplus\right.\right.$ $\left.\left.\mu_{2}\right)\left(x, 0_{V_{2}}\right),\left(\mu_{1} \oplus \mu_{2}\right)\left(y, 0_{V_{2}}\right)\right)=C\left(C\left(\mu_{1}(x), \mu_{2}\left(0_{V_{2}}\right)\right),\left(C\left(\mu_{1}(y), \mu_{2}\left(0_{V_{2}}\right)\right)\right)=C\left(\mu_{1}(x), \mu_{1}(y)\right)\right.$. Therefore, $\mu_{1}(x+y) \leq C\left(\mu_{1}(x), \mu_{1}(y)\right)$.

Also, since $\mu_{1}(x) \geq \mu_{2}\left(0_{V_{2}}\right)$, so $\mu_{1}(-x) \geq \mu_{2}\left(0_{V_{2}}\right)$ for all $x \in V_{1}$ and then $\mu_{1}(-x)=$ $C\left(\mu_{1}(-x), \mu_{2}\left(0_{V_{2}}\right)\right)=\left(\mu_{1} \oplus \mu_{2}\right)\left(-x, 0_{V_{2}}\right)=\left(\mu_{1} \oplus \mu_{2}\right)\left(-\left(x, 0_{V_{2}}\right)\right) \leq\left(\mu_{1} \oplus \mu_{2}\right)\left(x, 0_{V_{2}}\right)=$ $C\left(\mu_{1}(x), \mu_{2}\left(0_{V_{2}}\right)\right)=\mu_{1}(x)$.

Also, by using $\mu_{1}(x) \geq \mu_{2}\left(0_{V_{2}}\right)$, we get $\mu_{1}(a x) \geq \mu_{2}\left(0_{V_{2}}\right)$ for all $x \in V_{1}$ and $a \in \mathbb{F}$. Now $\mu_{1}(a x)=C\left(\mu_{1}(a x), \mu_{2}\left(0_{V_{2}}\right)\right)=\left(\mu_{1} \oplus \mu_{2}\right)\left(a x, 0_{V_{2}}\right)=\left(\mu_{1} \oplus \mu_{2}\right)\left(a\left(x, 0_{V_{2}}\right)\right) \leq\left(\mu_{1} \oplus \mu_{2}\right)\left(x, 0_{V_{2}}\right)=$ $C\left(\mu_{1}(x), \mu_{2}\left(0_{V_{2}}\right)\right)=\mu_{1}(x)$ therefore, $\mu_{1}(a x) \leq \mu_{1}(x)$. Hence $\mu_{1} \in A F C\left(V_{1}\right)$.

(2) The proof is similar as of (1).

(3) It is obvious.

Definition 11. Let $V$ be a space over field $\mathbb{F}, W$ be a subspace of $V$ and $\mu_{W}: W \rightarrow[0,1]$ be a fuzzy subspace. Define $\mu_{\frac{V}{W}}: \frac{V}{W} \rightarrow[0,1]$ as:

$$
\mu_{V}(x+W)=\left\{\begin{aligned}
C\left(\mu_{W}(x), \mu_{W}(w)\right) & \text { if } x \neq w \\
0 & \text { if } x=w
\end{aligned}\right.
$$

for all $x \in V$ and $w \in W$.

Theorem 24. In definition 11, if $C$ be idempotent t-conorm and $\mu_{W} \in A F C(W)$, then $\mu_{\frac{V}{W}} \in A F C\left(\frac{V}{W}\right)$.

Proof. Let $x+W, y+W \in \frac{V}{W}$ such that $x, y \neq w$ and $a \in \mathbb{F}$. Then

(1) Since $C$ is idempotent, so by Lemma 1 , we have

$$
\begin{aligned}
\mu_{\frac{V}{W}}((x+W)+(y+W)) & =\mu_{\frac{V}{W}}(x+y+W) \\
& =C\left(\mu_{W}(x+y), \mu_{W}(w)\right) \\
& \leq C\left(C\left(\mu_{W}(x), \mu_{W}(y)\right), \mu_{W}(w)\right) \\
& =C\left(C\left(\mu_{W}(x), \mu_{W}(y)\right), C\left(\mu_{W}(w), \mu_{W}(w)\right)\right) \\
& =C\left(C\left(\mu_{W}(x), \mu_{W}(w)\right), C\left(\mu_{W}(y), \mu_{W}(w)\right)\right) \\
& =C\left(\mu_{\frac{V}{W}}(x+W), \mu_{\frac{V}{W}}(y+W)\right) .
\end{aligned}
$$

(2) $\mu_{V}(-x+W)=C\left(\mu_{W}(-x), \mu_{W}(w)\right) \leq C\left(\mu_{W}(x), \mu_{W}(w)\right)=\mu_{V}(x+W)$.

(3) $\mu_{V}^{W}(a x+W)=C\left(\mu_{W}(a x), \mu_{W}(w)\right) \leq C\left(\mu_{W}(x), \mu_{W}(w)\right)=\mu_{V}^{W}(x+W)$.

Then $\mu_{V} \in \operatorname{AFC}\left(\frac{V}{W}\right)$.

Definition 12. Let $\mu \in A F C(V)$, we say that $\mu$ is normal if there exists $x \in V$ such that $\mu(x)=1$.

Note that if $\mu$ normal, then $\mu(0)=1$, hence $\mu$ is a normal if and only if $\mu(0)=1$. The set of all normal anti fuzzy subspaces of $V$ under $t$-conorm $C$ is denoted by $N A F C(V)$. 
Theorem 25. Let $\mu \in A F C(V)$ and $\hat{\mu}: V \rightarrow[0,1]$ be a fuzzy set defined by $\hat{\mu}(x)=\mu(x)+1-\mu(0)$ for all $x \in V$. Then $\hat{\mu} \in \operatorname{NFST}(V)$.

Proof. Let $x, y \in V$ and $a \in \mathbb{F}$. Then

(1) $\hat{\mu}(x+y)=\mu(x+y)+1-\mu(0) \leq C(\mu(x), \mu(y))+1-\mu(0)=C(\mu(x)+1-\mu(0), \mu(y)+1-\mu(0))$ $=C(\hat{\mu}(x), \hat{\mu}(y))$.

(2) $\hat{\mu}(-x)=\mu(-x)+1-\mu(0) \leq \mu(x)+1-\mu(0)=\hat{\mu}(x)$.

(3) $\hat{\mu}(a x)=\mu(a x)+1-\mu(0) \leq \mu(x)+1-\mu(0)=\hat{\mu}(x)$.

Thus $\hat{\mu} \in \operatorname{AFC}(V)$. Also, $\hat{\mu}(0)=\mu(0)+1-\mu(0)=1$. Therefore $\hat{\mu} \in \operatorname{NAFC}(V)$.

Theorem 26. Let $\mu \in A F C(V)$. Then $\mu \in N A F C(V)$ if and only if $\hat{\mu}=\mu$.

Proof. Let $\mu \in N A F C(V)$ then $\mu(0)=1$ and then $\hat{\mu}(x)=\mu(x)+1-\mu(0)=\mu(x)+1-1=\mu(x)$ for all $x \in V$. Conversely, let $\hat{\mu}=\mu$ and from Theorem 17 , we have that $\hat{\mu} \in \operatorname{NAFC}(V)$ and so $\mu \in \operatorname{NAFC}(V)$.

Theorem 27. Let $\mu \in A F C(V)$ and $\bar{\mu}: V \rightarrow[0,1]$ be a fuzzy set defined by $\bar{\mu}(x)=\frac{\mu(x)}{\mu(0)}$ for all $x \in V$ with $\mu(0) \neq 0$. Then $\bar{\mu} \in \operatorname{NAFC}(V)$.

Proof. Let $x, y \in V$ and $a \in \mathbb{F}$. Then

(1) $\bar{\mu}(x+y)=\frac{\mu(x+y)}{\mu(0)} \leq \frac{C(\mu(x), \mu(y))}{\mu(0)}=C\left(\frac{\mu(x)}{\mu(0)}, \frac{\mu(y)}{\mu(0)}\right)=C(\bar{\mu}(x), \bar{\mu}(y))$.

(2) $\bar{\mu}(-x)=\frac{\mu(-x)}{\mu(0)} \leq \frac{\mu(x)}{\mu(0)}=\bar{\mu}(x)$.

(3) $\bar{\mu}(a x)=\frac{\mu(a x)}{\mu(0)} \leq \frac{\mu(x)}{\mu(0)}=\bar{\mu}(x)$.

Thus $\bar{\mu} \in A F C(V)$. Also, $\bar{\mu}(0)=\frac{\mu(0)}{\mu(0)}=1$. Hence $\bar{\mu} \in \operatorname{NAFC}(V)$.

\section{Linear transformations over anti fuzzy vector spaces under $t$-conorms}

Definition 13. Let $f: V \rightarrow W$ be a linear transformation over the field $\mathbb{F}$. Let $\mu \in[0,1]^{V}$ and $v \in[0,1]^{W}$. Define $f(\mu) \in[0,1]^{W}$ and $f^{-1}(v) \in[0,1]^{V}$ as:

$$
f(\mu)(w)=\left\{\begin{aligned}
\inf \{\mu(v) \mid v \in V, f(v)=w\} & \text { if } f^{-1}(w) \neq \varnothing \\
0 & \text { if } f^{-1}(w)=\varnothing .
\end{aligned}\right.
$$

Also $f^{-1}(v)(v)=v(f(v))$.

Theorem 28. Let $f$ be an epimorphism linear transformation from vector space $V$ into vector space $W$ over field $\mathbb{F}$. If $\mu \in A F C(V)$, then $f(\mu) \in A F C(W)$.

Proof. (1) Let $w_{1}, w_{2} \in W$. Then

$$
\begin{aligned}
f(\mu)\left(w_{1}+w_{2}\right) & =\inf \left\{\mu\left(v_{1}+v_{2}\right) \mid v_{1}, v_{2} \in V, f\left(v_{1}\right)=w_{1}, f\left(v_{2}\right)=w_{2}\right\} \\
& \leq \inf \left\{C\left(\mu\left(v_{1}\right), \mu\left(v_{2}\right)\right) \mid v_{1}, v_{2} \in V, f\left(v_{1}\right)=w_{1}, f\left(v_{2}\right)=w_{2}\right\} \\
& =C\left(\inf \left\{\mu\left(v_{1}\right) \mid f\left(v_{1}\right)=w_{1}\right\}, \inf \left\{\mu\left(v_{2}\right) \mid f\left(v_{2}\right)=w_{2}\right\}\right) \\
& =C\left(f(\mu)\left(w_{1}\right), f(\mu)\left(w_{2}\right)\right) .
\end{aligned}
$$

(2)

$$
\begin{aligned}
f(\mu)(-w) & =\inf \{\mu(-v) \mid v \in V, f(-v)=-w\} \\
& =\inf \{\mu(-v) \mid v \in V,-f(v)=-w\} \\
& \leq \inf \{\mu(v) \mid v \in V, f(v)=w\}=f(\mu)(w) .
\end{aligned}
$$


(3) Let $v \in V$ and $a \in \mathbb{F}$. Now

$$
\begin{aligned}
f(\mu)(a w) & =\inf \{\mu(a v) \mid a v \in V, f(a v)=a w\} \\
& \leq \inf \{\mu(v) \mid v \in V, a f(v)=a w\} \\
& =\inf \{\mu(v) \mid v \in V, f(v)=w\} \\
& =f(\mu)(w) .
\end{aligned}
$$

Therefore $f(\mu) \in A F C(W)$.

Theorem 29. Let $f$ be an epimorphism linear transformation from vector space $V$ into vector space $W$ over field $\mathbb{F}$. If $\mu \in N A F C(V)$, then $f(\mu) \in N A F C(W)$.

Proof. By Theorem 28, we have $f(\mu) \in A F C(W)$. Suppose that $0_{V}$ and $0_{W}$ are the identity elements of $V$ and $W$, respectively. Since $\mu \in N A F C(V)$ so $\mu\left(0_{V}\right)=1$ and then

$$
\begin{aligned}
f(\mu)\left(0_{W}\right) & =\inf \left\{\mu\left(0_{V}\right) \mid 0_{V} \in V, f\left(0_{V}\right)=0_{W}\right\} \\
& =\inf \left\{1 \mid 0_{V} \in V, f\left(0_{V}\right)=0_{W}\right\} \\
& =1
\end{aligned}
$$

Hence $f(\mu) \in N A F C(W)$.

Theorem 30. Let $f$ be a linear transformation from vector space $V$ into vector space $W$ over field $\mathbb{F}$. If $v \in A F C(W)$, then $f^{-1}(v) \in A F C(V)$.

Proof. (1) Let $v_{1}, v_{2} \in V$. Then

$$
\begin{aligned}
f^{-1}(v)\left(v_{1}+v_{2}\right) & =v\left(f\left(v_{1}+v_{2}\right)\right) \\
& =v\left(f\left(v_{1}\right)+f\left(v_{2}\right)\right) \\
& \leq C\left(v \left(f\left(v_{1}\right), v\left(f\left(v_{2}\right)\right)\right.\right. \\
& =C\left(f^{-1}(v)\left(v_{1}\right), f^{-1}(v)\left(v_{2}\right)\right) .
\end{aligned}
$$

(2) Let $v \in V$. Then

$$
f^{-1}(v)(-v)=v(f(-v))=v(-f(v)) \leq v(f(v))=f^{-1}(v)(v) .
$$

(3) Let $v \in V$ and $a \in \mathbb{F}$. Then

$$
f^{-1}(v)(a v)=v(f(a v))=v(a f(v)) \leq v(f(v))=f^{-1}(v)(v) .
$$

Thus $f^{-1}(v) \in A F C(V)$.

Theorem 31. Let $f$ be a linear transformation from vector space $V$ into vector space $W$ over field $\mathbb{F}$. If $v \in N A F C(W)$, then $f^{-1}(v) \in N A F C(V)$.

Proof. Theorem 30 gives us $f^{-1}(v) \in A F C(V)$. Suppose that $0_{V}$ and $0_{W}$ be the identity elements of $V$ and $W$, respectively. As $v \in \operatorname{NAFC}(W)$ so $v\left(0_{W}\right)=1$ and then $f^{-1}(v)\left(0_{V}\right)=v\left(f\left(0_{V}\right)\right)=v\left(0_{W}\right)=1$. Thus $f^{-1}(v) \in \operatorname{NAFC}(V)$.

Acknowledgments: I would like to thank the reviewers for carefully reading the manuscript and making several helpful comments to increase the quality of the paper.

Conflicts of Interest: "The author declares no conflict of interest."

\section{References}

[1] Zadeh, L. A. (1965). Fuzzy sets. Information and Control, 8(3), 338-353.

[2] Rosenfeld, A. (1971). Fuzzy groups. Journal of Mathematical Analysis and Applications, 35(3), 512-517.

[3] Nanda, S. (1991). Fuzzy linear spaces over valued fields. Fuzzy Sets and Systems, 42(3), 351-354. 
[4] Katsaras, A. K., \& Liu, D. B. (1977). Fuzzy vector spaces and fuzzy topological vector spaces. Journal of Mathematical Analysis and Applications, 58(1), 135-146.

[5] Abdukhalikov, K. S. (1996). The dual of a fuzzy subspace. Fuzzy sets and systems, 82(3), 375-381.

[6] Lowen, R. (1980). Convex fuzzy sets. Fuzzy sets and Systems, 3(3), 291-310.

[7] Lubczonok, P. (1990). Fuzzy vector spaces. Fuzzy sets and systems, 38(3), 329-343.

[8] Lubczonok, G., \& Murali, V. (2002). On flags and fuzzy subspaces of vector spaces. Fuzzy sets and systems, 125(2), 201-207.

[9] Höhle, U. (1978). Probabilistic uniformization of fuzzy topologies. Fuzzy Sets and Systems, 1(4), 311-332.

[10] Alsina, C., Trillas, E., \& Valverde, L. (1983). On some logical connectives for fuzzy sets theory. Journal of Mathematical Analysis and Applications, 93(1), 15-26.

[11] Gupta, M. M., \& Qi, J. (1991). Theory of T-norms and fuzzy inference methods. Fuzzy sets and systems, 40(3), 431-450.

[12] Yandong, Y. (1985). Triangular norms and TNF-sigma-algebras. Fuzzy Sets and Systems, 16(3), 251-264.

[13] Rasuli, R. (2016). Fuzzy Ideals of Subtraction Semigroups with Respect to A t-norm and A t-conorm. The Journal of Fuzzy Mathematics Los Angeles, 24(4), 881-892.

[14] Rasuli, R. (2019). Fuzzy equivalence relation, fuzzy congrunce relation and fuzzy normal subgroups on group G over t-norms. Asian Journal of Fuzzy and Applied Mathematics, 7(2) 14-28.

[15] Rasuli, R. (2019). Norms over anti fuzzy G-submodules. MathLAB J, 2, 56-64.

[16] Rasuli, R. (2019). Norms over bifuzzy bi-ideals with operators in semigroups. Notes on Intuitionistic Fuzzy Sets, 25, $1-11$.

[17] Rasuli, R. (2019). Norms over basic operations on intuitionistic fuzzy sets. The Journal of Fuzzy Mathematics Los Angles, 27(3), 561-582.

[18] Rasuli, R. (2019). T-fuzzy bi-ideals in semirings. Earthline Journal of Mathematical Sciences, 2(1), 241-263.

[19] Enneth, Hoffman, H. \& R. Kunze, R. (1961). Linear Algebra. Prentice-Hall, Inc. , Englewood Cliffs, New Jersey.

[20] Malik, D. S. \& Mordeson, J.N. (1995). Fuzzy Commutative Algebra. World Science publishing Co. Pte. Ltd.

[21] Buckley, J. J. \& Eslami, E. (2002). An introduction to fuzzy logic and fuzzy sets. Springer-Verlag Berlin Heidelberg.

(C) 2020 by the authors; licensee PSRP, Lahore, Pakistan. This article is an open access article distributed under the terms and conditions of the Creative Commons Attribution (CC-BY) license (http://creativecommons.org/licenses/by/4.0/). 